\title{
Lymphocutaneous Sporotrichosis in a Patient with Underlying Systemic Lupus Erythematosus
}

\author{
Asrul Abdul Wahab, ${ }^{1}$ Chuan Hun Ding, ${ }^{1}$ Nurul Hafizah Mohd Yusoff ${ }^{2}$
}

\begin{abstract}
:
Sporotrichosis is a globally prevalent subcutaneous mycosis commonly acquired through traumatic inoculation. A 66-year-old woman with underlying systemic lupus erythematosus (SLE) presented initially with a painful non-healing nodule on her right thumb. There was no history of traumatic contact with soil, plants or animals. She was started on an antibiotic but developed multiple nodules on her right forearm which appeared to spread proximally. Culture of her skin biopsy specimen yielded Sporothrix schenckii. The lesions were successfully treated with oral itraconazole. In conclusion, a history of traumatic inoculation need not always be present in SLE patients with sporotrichosis and obtaining a laboratory diagnosis is crucial for those presenting with nodular skin lesions that spread proximally.
\end{abstract}

Key words: Sporothrix schenckii, sporotrichosis, systemic lupus erythematosus, itraconazole.

DOI: https://doi.org/l0.3329/jom.v2Iil.4410I

Copyright: (C) 2020 Wahab A A et al. This is an open access article published under the Creative Commons Attribution-NonCommercial-NoDerivatives 4.0 International License, which permits use, distribution and reproduction in any medium, provided the original work is properly cited, is not changed in any way and it is not used for commercial purposes.

Received: 17 December, 2018;

Accepted: 06 March, 2019

\section{Introduction:}

Sporotrichosis is an endemic mycosis caused by Sporothrix schenckii, a thermally dimorphic fungus that is prevalent worldwide but particularly common in tropical and subtropical areas. Typically, humans are infected following traumatic inoculation of the causative agent while engaging in outdoor activities such as farming, gardening and animal husbandry. ${ }^{1}$ Among the endemic mycoses, sporotrichosis is distinct owing to its potential for zoonotic transmission following scratches or bites from certain animals. ${ }^{1,2}$ The majority of sporotrichosis cases are limited to the skin and subcutaneous tissues, with dissemination to viscera or osteo-articular structures being rather rare. ${ }^{3}$ S. schenckii is a true pathogen which causes infections even in immunocompetent individuals, although the extent of the disease varies with the state of immunity. ${ }^{2}$ Diabetes mellitus,

1. Department of Medical Microbiology \& Immunology, Faculty of Medicine, Universiti Kebangsaan Malaysia, Kuala Lumpur, Malaysia

2. Department of Pathology, Hospital Duchess of Kent, Sandakan, Sabah, Malaysia

Corresponding author: Assoc. Prof. Dr. Chuan Hun Ding, Department of Medical Microbiology \& Immunology, Level 16, Preclinical Building, Faculty of Medicine, Universiti Kebangsaan Malaysia, 56000 Kuala Lumpur, Malaysia. Email: dingch@ ppukm.ukm.edu.my chronic obstructive airway diseases, HIV infection and alcoholism are among the known risk factors for sporotrichosis. ${ }^{2}$ However, at the time of writing, reports of systemic lupus erythematosus being a predisposing condition are scanty in the medical literature.

\section{Case summary:}

A 66-year-old lady with a background history of SLE presented to the Emergency Department of Universiti Kebangsaan Malaysia Medical Centre with a one-week history of a painful nodule on her right thumb. Prior to her presentation in our Emergency Department, she was prescribed with a course of oral cloxacillin from a local clinic, but this did not ameliorate her condition. She did not recall any history of trauma or foreign body inoculation prior to the onset of the nodule. On examination, she was afebrile and her vital signs were stable. There was a swelling on her right thumb measuring $0.5 \mathrm{~cm} \times 0.5 \mathrm{~cm}$ associated with purulent discharge. Areas of erythema were also seen on her right forearm. An incision and drainage (I\&D) of the swelling was performed and she was discharged home with oral ampicillinsulbactam.

However, a week later, she presented again with pain at the previous I\&D wound associated with seropurulent discharge. An X-ray of the right hand revealed no evidence 
of osteomyelitis. She was managed conservatively with daily dressings and was given an out-patient appointment in the orthopedic clinic. However, her right thumb became ulcerated and several other painful erythematous nodules erupted on her right forearm which seemed to extend proximally. Thus, she was referred to the dermatologist who performed a punch skin biopsy of the nodules. The specimens were sent for microbiological culture and histopathological examination (HPE). The microbiological culture result from the skin biopsy tissues grew S. schenckii (Fig. 1). However, the HPE results were non-specific, with only lymphocytic infiltration at the dermal layer with extension to the dermis being noted. There were no granulomata, acid-fast bacilli or fungal elements seen.

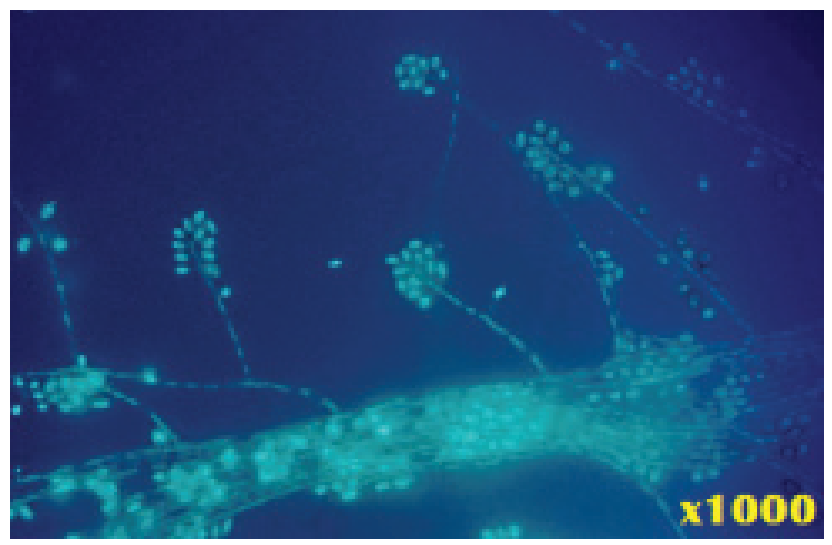

Figure 1: Phase contrast microscopy of the mold phase of Sporothrix schenckii showing one-celled conidia arranged sympodially at the tip of conidiophores, giving rise to a flower bouquet or daisy head arrangement.

A definitive diagnosis of sporotrichosis was made and she was started on oral itraconazole $200 \mathrm{mg}$ daily. During her follow up a month later, the ulcer over the right thumb showed evidence of healing and the multiple nodules on the right forearm were distinctively less erythematous. No new skin lesions were reported. The patient was told to continue the itraconazole therapy until 2 weeks after all her skin lesions had resolved.

\section{Discussion:}

Sporotrichosis has been traditionally dubbed "rose gardeners' disease" due to its association with individuals who cultivate roses. Infections are most often sporadic and follow traumatic inoculation sustained during the course of outdoor activities. ${ }^{3}$ The infection may also become established following scratches or bites from various domestic as well as wild animals such as dogs, cats, mice, armadillos and squirrels. ${ }^{2}$ Some infections have also been reported in laboratory personnel handling cultures of $S$. schenckii. ${ }^{4}$ Although in the majority of cases the infection is secondary to traumatic inoculation via scratches, pricks and other minor injuries, a history of trauma may not necessary be present. ${ }^{5}$ Similarly, our patient did not have a history of trauma or penetrating injury. Although monocytes/ macrophages play a key role in the host defence against sporotrichosis, the precise mechanisms of resistance and/or susceptibility to $S$. schenckii infections are unknown. ${ }^{6}$ Likewise, it is also uncertain if her underlying SLE increased her odds of contracting the disease due to the paucity of published data.

Following the inoculation of $S$. schenckii conidia into the skin or subcutaneous tissue, a primary lesion forms within days to weeks, initially as a papule but later enlarging to become a nodule which frequently ulcerates. Disease progression is typified by nodular lesions which erupt along the lymphatic distribution proximal to the initial lesion and lymphangitic streaks in between the nodules. ${ }^{2}$ The differential diagnoses of this form of sporotrichosis include sarcoidosis, mycobacterial infections, nocardiosis, chromoblastomycosis, blastomycosis, para-coccidioidomycosis, and leishmaniasis .$^{2,4}$ To further complicate matters, a small number of patients do not manifest the typical lymphangitic spread but instead present with fixed cutaneous lesions which remain at the inoculation site. ${ }^{7}$ Thus, obtaining a definitive diagnosis cannot be overemphasised because some of the conditions are either non-infectious in nature (i.e. sarcoidosis) or caused by bacteria which would not respond favourably to antifungal agents.

Sporotrichosis is diagnosable by correlating clinical or epidemiological data with laboratory findings. Culturing $S$. schenckii is still the gold standard to validate a clinical suspicion of sporotrichosis. ${ }^{3}$ Culture material from cutaneous lesions can either be aspirated, swabbed or obtained via tissue biopsy. The specimen can be cultured on Sabouraud dextrose agar containing chloramphenicol or on mycobiotic agar at room temperature $\left(25^{\circ} \mathrm{C}\right)$ to facilitate the growth of the mold phase of $S$. schenckii. ${ }^{5}$ To positively identify $S$. schenckii, conversion to the yeast phase when the mould is grown on enriched media (e.g. brain heart infusion agar) at body temperature $\left(35-37^{\circ} \mathrm{C}\right)$ has to be demonstrated. ${ }^{5}$ However, a tentative identification can already be made once the characteristic conidia formation is seen during the mold phase. ${ }^{3}$ Characteristically, the mold form of S. schenckii produces one-celled conidia in groups on small clustered denticles, resembling a flower bouquet. ${ }^{2,5}$ 
Nevertheless, culture is time consuming and can take up to two weeks to complete because the initial culture must be followed by demonstration of thermal dimorphism. Although more rapid, serological testing has not been found to be valuable in diagnosing sporotrichosis and is also not widely available. ${ }^{5}$ However, antibody detection may have a role in systemic manifestations or atypical forms of sporotrichosis and may be of value in monitoring response to treatment or detecting relapses. ${ }^{8}$ Molecular detection is another rapid diagnostic modality for sporotrichosis and is of value in culture-negative cases due to low fungal burden or secondary infections. However, molecular techniques for detecting $S$. schenckii DNA from clinical samples are scarce and commercial in-vitro diagnostic (IVD) kits are not widely available. ${ }^{5}$ Histopathological examination of biopsied specimens is also limited by the scantiness of fungal elements in the tissue (as was the case with this patient), being present in only 18 to $35.3 \%$ of cases. ${ }^{8}$

The treatment of lymphocutaneous sporotrichosis is relatively straightforward using oral chemotherapy with high success rates and few recurrences. Although there are numerous oral drugs which can be utilized to treat sporotrichosis, the two agents which are most widely used are potassium iodide (KI) and itraconazole, with the former being the treatment of choice in developing nations. ${ }^{9}$ While the usual dosage of itraconazole is $200 \mathrm{mg}$ per day, it can be increased up to $200 \mathrm{mg}$ twice daily if the clinical response is poor. ${ }^{3}$ Itraconazole should be administered until 2-4 weeks after all lesions have resolved, with a total treatment duration of 3-6 months. ${ }^{3}$ As for KI, the exact dosage is not established although a daily dose of 3-6 $\mathrm{g}$ has been recommended. ${ }^{9}$ Monotherapy with either KI or itraconazole is usually already sufficient to bring about clinical cure, although there are various combination therapy regimes (which may also include physical therapy such as cryo- or thermotherapy) that have been reported to reduce the total treatment duration. ${ }^{9}$

\section{Conclusions:}

This case demonstrates the delay in the diagnosis of lymphocutaneous sporothricosis due to lack of clinical suspicion. A typical history of traumatic inoculation need not always be present and although this is a case of sporotrichosis in an SLE patient, it remains to be elucidated if SLE is a predisposing factor. The role of the clinical microbiology laboratory in providing a definitive diagnosis of sporotrichosis cannot be overemphasised and all patients with nodular skin lesions that appear to spread proximally should have their lesions cultured.

\section{Conflict of interest: None.}

\section{Acknowledgement:}

The authors would like to thank the Dean of the Faculty of Medicine, Universiti Kebangsaan Malaysia for her support and permission to publish this article.

\section{References:}

1. Chakrabarti A, Bonifaz A, Gutierrez-Galhardo MC, Mochizuki T, Li S. Global epidemiology of sporotrichosis. Med Mycol. 2015;53(1):3-14.

2. Kauffman CA. Sporotrichosis. Clin Infect Dis. 1999;29:231-7.

3. Kauffman CA, Bustamante B, Chapman SW, Pappas PG. Clinical practice guidelines for the management of sporotrichosis: 2007 update by the Infectious Diseases Society of America. Clin Infect Dis. 2007;45:1255-65.

4. Cooper CR, Dixon DM, Salkin IF. Laboratory-acquired sporotrichosis. J Med Vet Mycol. 1992;30:169-71.

5. Barros MB, de Almeida Paes RA, Schubach AO. Sporothrix schenckii and sporotrichosis. Clin Microbiol Rev. 2011;24(4):633-54.

6. Carlos IZ, Sassá MF, da Graça Sgarbi DB, Placeres MC, Maia DC. Current research on the immune response to experimental sporotrichosis. Mycopathologia. 2009;168(1):1-10.

7. Kwon-Chung K. Comparison of isolates of Sporothrix schenckii obtained from fixed cutaneous lesions with isolates from other types of lesions. J Infect. 1979;139:424-31.

8. Orofino-Costa R, Macedo PM, Rodrigues AM, BernardesEngemann AR. Sporotrichosis: an update on epidemiology, etiopathogenesis, laboratory and clinical therapeutics. An Bras Dermatol. 2017;92(5):606-20.

9. Bonifaz A, Vázquez-González D. Diagnosis and treatment of lymphocutaneous sporotrichosis: What are the options? Curr Fungal Infect Rep. 2013;7(3):252-9. 